\title{
DIFFERENTIAL EQUATIONS AND FOLDING OF $n$-MANIFOLDS
}

\author{
I. MOUSA AND F. SALAMA
}

\begin{abstract}
In this paper we will describe some topological and geometric characters of $n$-manifold by using the properties of differential equations. The folding and unfolding of $n$-manifold into itself will be deduced from viewpoint of the differential equations.
\end{abstract}

Definition 1. An $n$-dimensional manifold is a Housdorff space such that each point has an open neighbourhood homeomorphic to the open $n$-dimensional disc $\cup^{n}(=\{x \in$ $\left.\left.\mathcal{R}^{n}:|x|<1\right\}\right)[10,14]$.

Definition 2. The set is compact if it closed and bounded [10].

Definition 3. For Riemannian manifolds $M$ and $N$ (not necessarily of the same dimension), a map $f: M \rightarrow N$ is said to be a "topological folding" of $M$ into $N$ if, for each piecewise geodesic path $\gamma: I \rightarrow M(I=[0,1] \subseteq R)$, the induced path $f \circ \gamma: I \rightarrow N$ is piecewise geodesic. If, in addition, $f: M \rightarrow N$ preserves lengths of paths, we call $f$ an "isometric folding" of $M$ into $N$. Thus an isometric folding is necessarily a topological folding. Some types of folding of manifolds discussed in $[3,6]$ and some applications of foldings introduced in [8].

Definition 4. Let $M$ and $N$ be two Riemannian manifolds of the same dimensions, a map $g: M \rightarrow N$ is said to be an "unfolding" of $M$ into $N$ if, for every piecwise geodesic path $\gamma: I \rightarrow M(I=[0,1] \subseteq R)$, the induced path $\gamma^{\prime}=g \circ \gamma: I \rightarrow N$ is piecewise geodesic but with length greater than that for $\gamma$.

i.e.

$$
\forall x, y \in M \Rightarrow d(x, y) \leq d(g(x), g(y))[2,4,5] .
$$

Theorem 1. If the eigenvalues $\lambda_{1}, \lambda_{2}, \ldots, \lambda_{n}$ of an $n \times n$ matrix $A$ are real and distinct, then any set of corresponding eigenvectors $\left\{V_{1}, V_{2}, \ldots, V_{n}\right\}$ forms a basis for $\mathcal{R}^{n}$, the matrix

$$
p=\left[V_{1} V_{2} \cdots V_{n}\right] \text { is invertible, } p^{-1} A p=\operatorname{diag}\left[\lambda_{1}, \lambda_{2}, \ldots, \lambda_{n}\right][12,13] .
$$

Received and revised February 16, 2004.

2000 Mathematics Subject Classification. 51H10, 57N10.

Key words and phrases. Differential equations, manifolds. 
Theorem 2. If the $2 n \times 2 n$ real matrix $A$ has $2 n$ distinct complex eigenvalues $\lambda_{j}=$ $a_{j}+i b_{j}$ and $\bar{\lambda}_{j}=a_{j}-i b_{j}$ and corresponding complex eigenvectors $w_{j}=u_{j}+i V_{j}$ and $\bar{w}_{j}=u_{j}-i V_{j}, j=1, \ldots, n$, then $\left\{u_{1} V_{1}, \ldots, u_{n} V_{n}\right\}$ is a basis for $\mathcal{R}^{2 n}$ the matrix $p=$ $\left[u_{1} V_{1}, \ldots, u_{n}, V_{n}\right]$ is invertible and

$$
p^{-1} A p=\operatorname{diag}\left[\begin{array}{cc}
a_{j} & -b_{j} \\
b_{j} & a_{j}
\end{array}\right]
$$

$A$ real $2 n \times 2 n$ matrix with $2 \times 2$ blocks along the diagonal.

\section{The main results.}

In this article we restrict on surfaces which represent 2-manifold and some examples of $n$-manifolds.

Theorem 3. The folding of any $n$-manifold represented by $D(V)=A V$ is a restriction on the elements of $n \times n$ matrix $A=\left[\begin{array}{cccc}\lambda_{1} & 0 & \cdots & 0 \\ 0 & \lambda_{2} & \cdots & 0 \\ \vdots & \vdots & \ddots & \vdots \\ 0 & 0 & \cdots & \lambda_{n}\end{array}\right]$ or on $\lambda_{1}, \lambda_{2}, \ldots, \lambda_{n}$, where $\lambda_{1}, \lambda_{2}, \ldots, \lambda_{n}$ are real (complex) and distinct eignevalues of $n \times n$ matrix $A, V$ is vector in $R^{n}$ and $\left|\lambda_{i}\right|<1$.

Proof. Let

$$
\text { i.e. } \quad \dot{V}=A V
$$

let $V=\left(x_{1}, x_{2}, \ldots, x_{n}\right)$, then $A=\left[\begin{array}{cccc}a_{11} & a_{12} & \cdots & a_{1 n} \\ a_{21} & a_{22} & \cdots & a_{2 n} \\ \vdots & \vdots & \ddots & \vdots \\ a_{n 1} & a_{n 2} & \cdots & a_{n n}\end{array}\right]$

the equation (1) can be written in the following form

$$
\left[\begin{array}{c}
x_{1} \\
x_{2} \\
\vdots \\
x_{n}
\end{array}\right]=\left[\begin{array}{cccc}
a_{11} & a_{12} & \cdots & a_{1 n} \\
a_{21} & a_{22} & \cdots & a_{2 n} \\
\vdots & \vdots & \ddots & \vdots \\
a_{n 1} & a_{n 2} & \cdots & a_{n n}
\end{array}\right]\left[\begin{array}{c}
x_{1} \\
x_{2} \\
\vdots \\
x_{n}
\end{array}\right]
$$

from Theorems 1 and 2 (according to $\lambda_{1}, \lambda_{2}, \ldots, \lambda_{n}$ are real or complex) we have the following system equivalent to the system (2)

$$
\left[\begin{array}{c}
y_{1} \\
y_{2} \\
\vdots \\
y_{n}
\end{array}\right]=\left[\begin{array}{cccc}
b_{1} & 0 & \cdots & 0 \\
0 & b_{2} & \cdots & 0 \\
\vdots & \vdots & \ddots & \vdots \\
0 & 0 & \cdots & b_{n}
\end{array}\right]\left[\begin{array}{c}
y_{1} \\
y_{2} \\
\vdots \\
y_{n}
\end{array}\right]
$$


and any $\boldsymbol{n} \times \boldsymbol{n}$ matrix equivalent to the matrix

$$
B=\left[\begin{array}{cccc}
b_{1} & 0 & \cdots & 0 \\
0 & b_{2} & \cdots & 0 \\
\vdots & \vdots & \ddots & \vdots \\
0 & 0 & \cdots & b_{n}
\end{array}\right]
$$

If $\left|b_{1}\right| \leq 1,\left|b_{2}\right| \leq 1, \ldots,\left|b_{n}\right| \leq 1$ then all vectors $\left(y_{1}, y_{2}, \ldots, y_{n}\right)$ folded.

Also

$$
\ddot{Y}=B \dot{Y}=B^{2} Y=\left[\begin{array}{cccc}
b_{1} & 0 & \cdots & 0 \\
0 & b_{2} & \cdots & 0 \\
\vdots & \vdots & \ddots & \vdots \\
0 & 0 & \cdots & b_{n}
\end{array}\right]\left[\begin{array}{c}
\dot{y}_{1} \\
\dot{y}_{2} \\
\vdots \\
\dot{y}_{n}
\end{array}\right]=\left[\begin{array}{cccc}
b_{1}^{2} & 0 & \cdots & 0 \\
0 & b_{2}^{2} & \cdots & 0 \\
\vdots & \vdots & \ddots & \vdots \\
0 & 0 & \cdots & b_{n}^{2}
\end{array}\right]\left[\begin{array}{c}
y_{1} \\
y_{2} \\
\vdots \\
y_{n}
\end{array}\right]
$$

if $\left|b_{1}\right|,\left|b_{2}\right|, \ldots,\left|b_{n}\right|<1$ then $\ddot{Y}$ represent the folding of $Y$

$$
\text { also } \begin{gathered}
\dddot{Y}=B^{3} Y=\left[\begin{array}{cccc}
b_{1}^{3} & 0 & \cdots & 0 \\
0 & b_{2}^{3} & \cdots & 0 \\
\vdots & \vdots & \ddots & \vdots \\
0 & 0 & \cdots & b_{n}^{3}
\end{array}\right]\left[\begin{array}{c}
y_{1} \\
y_{2} \\
\vdots \\
y_{n}
\end{array}\right] \\
\vdots \\
Y^{(n)}=B^{n} Y=\left[\begin{array}{cccc}
b_{1}^{n} & 0 & \cdots & 0 \\
0 & b_{2}^{n} & \cdots & 0 \\
\vdots & \vdots & \ddots & \vdots \\
0 & 0 & \cdots & b_{n}^{n}
\end{array}\right]\left[\begin{array}{c}
y_{1} \\
y_{2} \\
\vdots \\
y_{n}
\end{array}\right]
\end{gathered}
$$

this is a sequence of foldings $f_{n}$ of $Y$ which also is foldings of $X$.

Theorem 4. Let $X_{1}, X_{2}, \ldots, X_{n}$ are functions of $w_{1}, w_{2}, \ldots, w_{n}$, i.e. $X_{i}=X_{i}\left(w_{1}\right.$, $\left.w_{2}, \ldots, w_{n}\right), i \in\{1, \ldots, n\}$ and $X_{i}=W_{1 i}\left(w_{1}\right) W_{2 i}\left(w_{2}\right) \cdots W_{n i}\left(w_{n}\right)$ then the folding of any $n$-manifold represented by $f(X)=D_{w j} X=A X$, where $X=\left[\begin{array}{c}X_{1} \\ X_{2} \\ \vdots \\ X_{n}\end{array}\right]$ and $j \in\{1, \ldots, n\}$, is a restraction on the elements of $n \times n$ matrix $A=\left[\begin{array}{cccc}\lambda_{1} & 0 & \cdots & 0 \\ 0 & \lambda_{2} & \cdots & 0 \\ \vdots & \vdots & \ddots & \vdots \\ 0 & 0 & \cdots & \lambda_{n}\end{array}\right]$ or on the distinct and real (complex) eigenvalues $\lambda_{1}, \lambda_{2}, \ldots, \lambda_{n}$ of the $n \times n$ matrix $A$.

Proof. Let $X_{1}, X_{2}, \ldots, X_{n}$ are functions of $w_{1}, w_{2}, \ldots, w_{n}$, i.e. $X_{i}=X_{i}\left(w_{1}, w_{2}, \ldots\right.$, $\left.w_{n}\right), i \in\{1, \ldots, n\}$ and $X_{i}=W_{1 i}\left(w_{1}\right) W_{2 i}\left(w_{2}\right) \cdots W_{n i}\left(w_{n}\right), f: M \rightarrow M$ be a folding 
where $f \equiv D_{w j}$. Let we have the system

$$
D_{w j} X=A X
$$

where $\quad X=\left[\begin{array}{c}X_{1} \\ X_{2} \\ \vdots \\ X_{n}\end{array}\right], \quad A=\left[\begin{array}{cccc}a_{11} & a_{12} & \cdots & a_{1 n} \\ a_{21} & a_{22} & \cdots & a_{2 n} \\ \vdots & \vdots & \ddots & \vdots \\ a_{n 1} & a_{n 2} & \cdots & a_{n n}\end{array}\right]$

the system (1) can be written in the form

$$
\begin{aligned}
& D_{w j}\left[\begin{array}{c}
W_{11}\left(w_{1}\right) W_{21}\left(w_{2}\right) \cdots W_{n 1}\left(w_{n}\right) \\
W_{12}\left(w_{1}\right) W_{22}\left(w_{2}\right) \cdots W_{n 2}\left(w_{n}\right) \\
\vdots \\
W_{1 n}\left(w_{1}\right) W_{2 n}\left(w_{2}\right) \cdots W_{n n}\left(w_{n}\right)
\end{array}\right]
\end{aligned}
$$

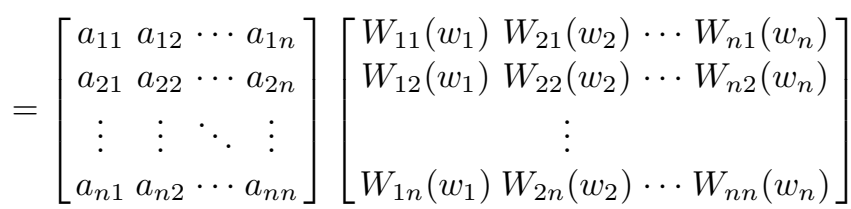

the above system can be reduced to the form

$$
\left[\begin{array}{c}
\dot{W}_{j 1} \\
\dot{W}_{j 2} \\
\vdots \\
\dot{W}_{j n}
\end{array}\right]=\left[\begin{array}{cccc}
a_{11} & a_{12} & \cdots & a_{1 n} \\
a_{21} & a_{22} & \cdots & a_{2 n} \\
\vdots & \vdots & \ddots & \vdots \\
a_{n 1} & a_{n 2} & \cdots & a_{n n}
\end{array}\right]\left[\begin{array}{c}
W_{j 1} \\
W_{j 2} \\
\vdots \\
W_{j n}
\end{array}\right]
$$

from theorem (3) we find that the folding of any $n$-manifold into itself represented by the system (2) is a restriction on the elements of the matrix $A=\left[\begin{array}{cccc}\lambda_{1} & 0 & \cdots & 0 \\ 0 & \lambda_{2} & \cdots & 0 \\ \vdots & \vdots & \ddots & \vdots \\ 0 & 0 & \cdots & \lambda_{n}\end{array}\right]$ or on the distinct and real (complex) eigenvalues $\lambda_{1}, \lambda_{2}, \ldots, \lambda_{n}$ of the $n \times n$ matrix $A$. Then also the folding of any $n$-manifold represented by $D_{w j} X=A X$ is a restriction on the elements of the matrix $A=\left[\begin{array}{cccc}\lambda_{1} & 0 & \cdots & 0 \\ 0 & \lambda_{2} & \cdots & 0 \\ \vdots & \vdots & \ddots & \vdots \\ 0 & 0 & \cdots & \lambda_{n}\end{array}\right]$ or on the distinct and real (complex) eigenvalues $\lambda_{1}, \lambda_{2}, \ldots, \lambda_{n}$ of the $n \times n$ matrix $A$.

Corollary. The limit of the foldings of the conditional folding in Theorem 3 is a manifold of dimension $k, k<n$. 
Proof. Let $D^{m} Y=f_{m}(Y), m=1,2, \ldots$, we have a sequence $f_{1}, f_{2}, \ldots, f_{m}$.

Then $\quad D^{m} Y=\left[\begin{array}{cccc}b_{1}^{m} & 0 & \cdots & 0 \\ 0 & b_{2}^{m} & \cdots & 0 \\ \vdots & \vdots & \ddots & \vdots \\ 0 & 0 & \cdots & b_{n}^{m}\end{array}\right]\left[\begin{array}{c}y_{1} \\ y_{2} \\ \vdots \\ y_{n}\end{array}\right]=\left[\begin{array}{c}b_{1}^{m} y_{1} \\ b_{2}^{m} y_{2} \\ \vdots \\ b_{n}^{m} y_{n}\end{array}\right]=\left(b_{1}^{m} y_{1}, b_{2}^{m} y_{2}, \ldots, b_{n}^{m} y_{n}\right)$.

Such that $\left|b_{i}^{m}\right|<<1, \forall i \in\{1, \ldots, n\}$ also $\left|b_{k}^{m}\right|<<1$ where $k \in\{1, \ldots, n\}$. Let $b_{j} \rightarrow 0$ as $m \rightarrow \infty$ then $\lim _{m \rightarrow \infty} f_{m}(Y)=\left(\xi_{1} y_{1}, \xi_{2} y_{2}, \ldots, 0, \ldots, \xi_{n} y_{n}\right) \in R^{n-1}$, where $b_{j}<<<b_{i}$, $\forall i \in\{1, \ldots, n\}$ and $\lim _{m \rightarrow \infty} b_{j}^{m} \rightarrow 0$ faster than the other $b_{i}, i \neq j$. Then the manifold must be of dimension $k$ such that $\max k=n-1$. See Figure 1 .
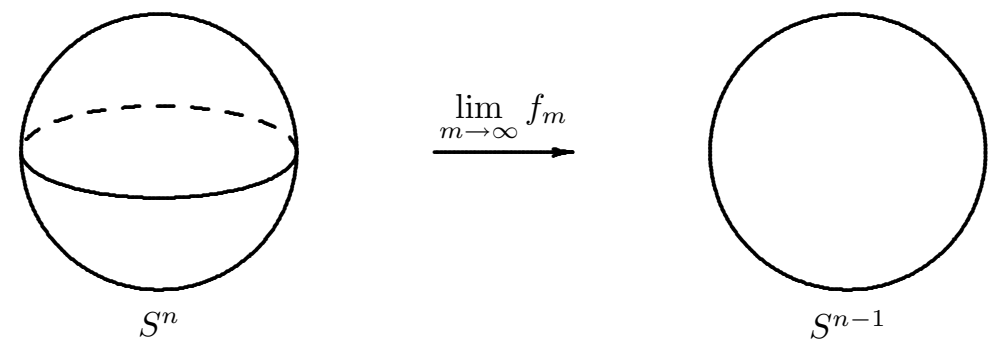

Figure 1.

Corollary. The end of the limit of foldings in Theorem 3 is a 0-manifold.

Proof.

Let $\quad f_{m}(Y)=D^{m} Y=\left[\begin{array}{cccc}b_{1}^{m} & 0 & \cdots & 0 \\ 0 & b_{2}^{m} & \cdots & 0 \\ \vdots & \vdots & \ddots & \vdots \\ 0 & 0 & \cdots & b_{n}^{m}\end{array}\right]\left[\begin{array}{c}y_{1} \\ y_{2} \\ \vdots \\ y_{n}\end{array}\right]=\left[\begin{array}{c}b_{1}^{m} y_{1} \\ b_{2}^{m} y_{2} \\ \vdots \\ b_{n}^{m} y_{n}\end{array}\right]=\left(b_{1}^{m} y_{1}, b_{2}^{m} y_{2}, \ldots, b_{n}^{m} y_{n}\right)$

$$
\begin{gathered}
\text { Since } \lim _{m \rightarrow \infty} f_{m}(Y)=\left(\xi_{1} y_{1}, \xi_{2} y_{2}, \ldots, 0, \xi_{k+1} y_{k+1}, \ldots, \xi_{n} y_{n}\right) \\
f_{m 1}=\lim _{m \rightarrow \infty} f_{m}(Y)=\left(\xi_{1} y_{1}, \ldots, 0, \xi_{k+1} y_{k+1}, \ldots, \xi_{n} y_{n}\right), \\
f_{m 2}=\left(\xi_{1} y_{1}, \ldots, 0,0, \xi_{k+1} y_{k+1}, \ldots, \xi_{n} y_{n}\right) \\
\vdots \\
\vdots \\
\lim _{k \rightarrow \infty} f_{m k}(y)=(0,0, \ldots, 0) \quad \text { which is 0-manifold (See Figure 2). }
\end{gathered}
$$




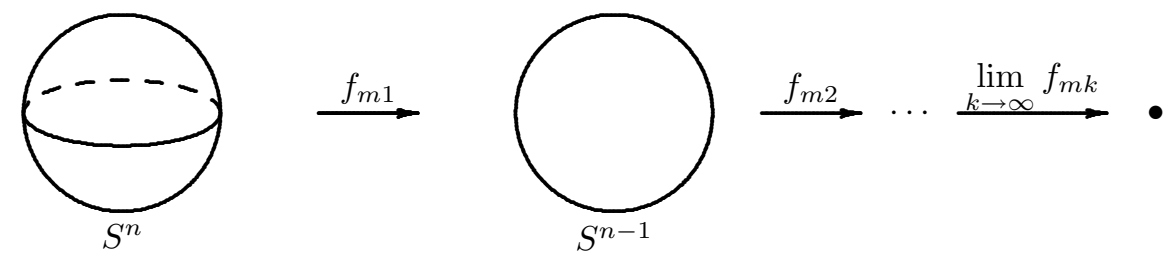

Figure 2.

From the above theorem, the two types of differentiations $X_{\theta}, X_{\phi}$, where $X=$ $(x(\theta, \phi), y(\theta, \phi), z(\theta, \phi))$, the first is a limit of foldings, the second is a folding. The first type induces a limit of foldings of the tangent space, the second type induces a folding of the tangent space.

Example 1. The parametric equations of the unit sphere $S^{2}$ given by

$$
\begin{aligned}
& x=\cos \theta \sin \phi \\
& y=\sin \theta \sin \phi \\
& z=\cos \phi
\end{aligned}
$$

where $0<\theta \leq 2 \pi$ and $0 \leq \phi \leq \pi$ by differentiating the above system with respect to $\theta$ and $\phi$ we have the boundary-value partial differential equations of the unit sphere $S^{2}$.

$$
\begin{aligned}
x_{\theta} & =-y \quad 0<\theta \leq 2 \pi, 0 \leq \phi \leq \pi \\
y_{\theta} & =x \\
z_{\theta} & =0 \\
x_{\phi} & =\cos \theta \sin \phi \\
y_{\phi} & =\sin \theta \sin \phi \\
z_{\phi} & =-\sin \phi
\end{aligned}
$$

the boundary condations are

$$
\begin{aligned}
& x(\theta, 0)=x(\theta, \pi)=0 \quad 0<\theta \leq 2 \pi \\
& y(\theta, 0)=y(\theta, \pi)=0 \\
& z(\theta, 0)=1, \quad z(\theta, \pi)=-1
\end{aligned}
$$

if we take the part

$$
X_{\theta}=\left[\begin{array}{ccc}
0 & -1 & 0 \\
1 & 0 & 0 \\
0 & 0 & 0
\end{array}\right] X
$$


we find the eigenvalues of the matrix $\left[\begin{array}{ccc}0 & -1 & 0 \\ 1 & 0 & 0 \\ 0 & 0 & 0\end{array}\right]$ are $\lambda_{1,2}= \pm i$ and $\lambda_{3}=0$ if we take $\left|\lambda_{1}\right|<1,\left|\lambda_{2}\right|<1$ we have a limit folding of the unit sphere $S^{2}$ and its tangent space (see Figure 3).
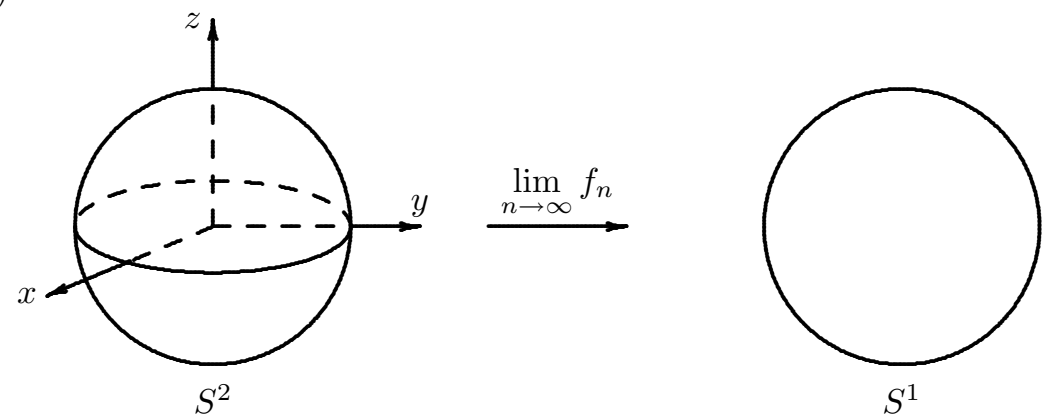

Figure 3.

A restriction of the boundary conditions in the boundary - value partial differential equations and the singularity of the folding at this restriction also making folding. The following example show this idea,

Example 2. From Example 1 by doing restriction of the boundary conditions we will have many cases of foldings of the unit sphere $S^{2}$. Now we will show those cases,

Case 1. If we take the boundary conditions in the form

$$
\begin{array}{rlrl}
x\left(\theta, \frac{\pi}{4}\right) & =\frac{1}{\sqrt{2}} \cos \theta, & x\left(\theta, \frac{3 \pi}{4}\right) & =\frac{1}{\sqrt{2}} \cos \theta, \\
y\left(\theta, \frac{\pi}{4}\right) & =\frac{1}{\sqrt{2}} \sin \theta, & y\left(\theta, \frac{3 \pi}{4}\right) & =\frac{1}{\sqrt{2}} \sin \theta, \\
z\left(\theta, \frac{\pi}{4}\right) & =\frac{1}{\sqrt{2}}, & z\left(\theta, \frac{3 \pi}{4}\right) & =\frac{-1}{\sqrt{2}}, \\
0 & <\theta \leq 2 \pi, & \frac{\pi}{4} \leq \phi \leq \frac{3 \pi}{4},
\end{array}
$$
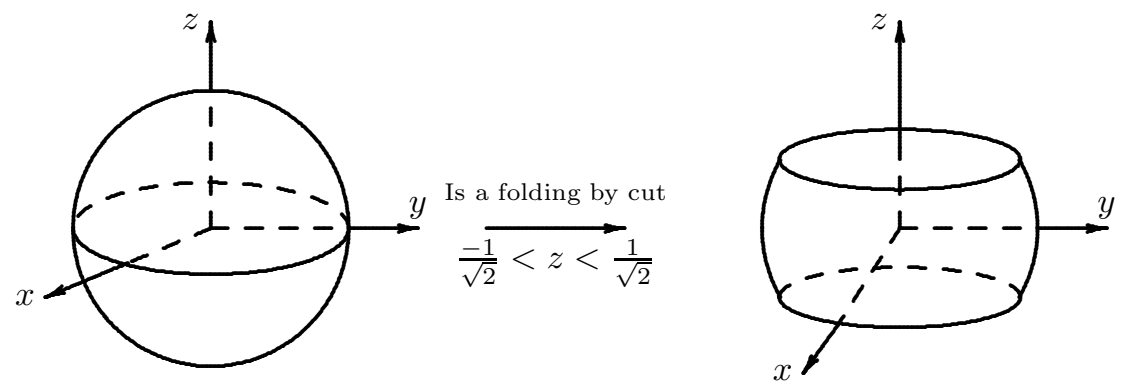
the result sphere is 2-manifold with boundary.

Case 2. If we change the boundary conditions into

$$
\begin{array}{rlrl}
x\left(\theta, \frac{\pi}{4}\right) & =\frac{1}{\sqrt{2}} \cos \theta, & x(\theta, \pi) & =0, \\
y\left(\theta, \frac{\pi}{4}\right) & =\frac{1}{\sqrt{2}} \sin \theta, & y(\theta, \pi) & =0, \\
z\left(\theta, \frac{\pi}{4}\right) & =\frac{1}{\sqrt{2}}, & z(\theta, \pi) & =-1, \\
0 & <\theta \leq 2 \pi, & \frac{\pi}{4} \leq \phi \leq \pi,
\end{array}
$$
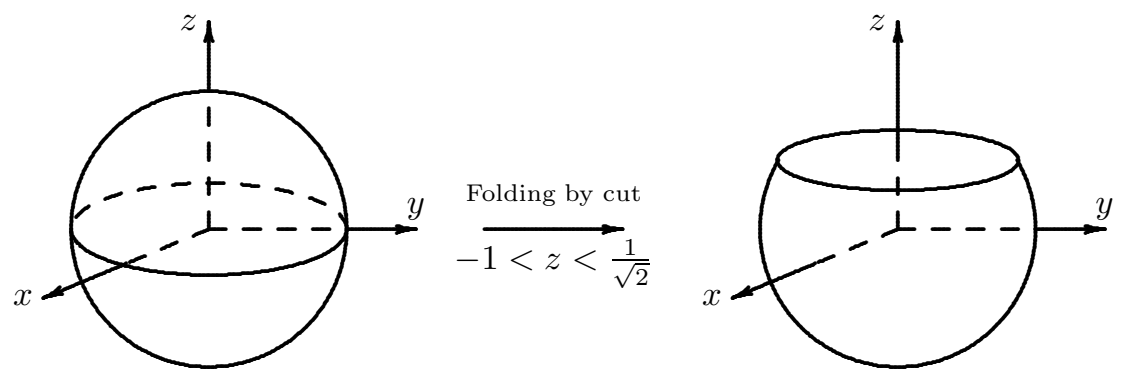

the graph after folding is 2-manifold

Case 3. If the boundary conditions are

$$
\begin{array}{ll}
x\left(\theta, \frac{\pi}{2}\right)=\cos \theta, & x(\theta, \pi)=0, \\
y\left(\theta, \frac{\pi}{2}\right)=\sin \theta, & y(\theta, \pi)=0, \\
z\left(\theta, \frac{\pi}{2}\right)=0, & z(\theta, \pi)=-1, \\
0<\theta \leq 2 \pi, & \frac{\pi}{2} \leq \phi \leq \pi,
\end{array}
$$
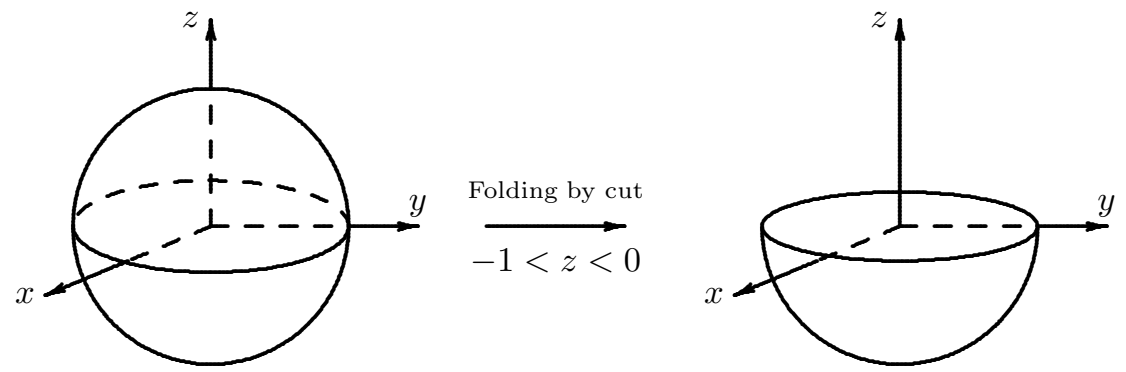
Case 4. Consider the boundary conditions on the form

$$
\begin{array}{ll}
x(\theta, 0)=0, & x\left(\theta, \frac{\pi}{2}\right)=\cos \theta, \\
y(\theta, 0)=0, & y\left(\theta, \frac{\pi}{2}\right)=\sin \theta, \\
z(\theta, 0)=1, & z\left(\theta, \frac{\pi}{2}\right)=0, \\
0<\theta \leq 2 \pi, & 0 \leq \phi \leq \frac{\pi}{2},
\end{array}
$$
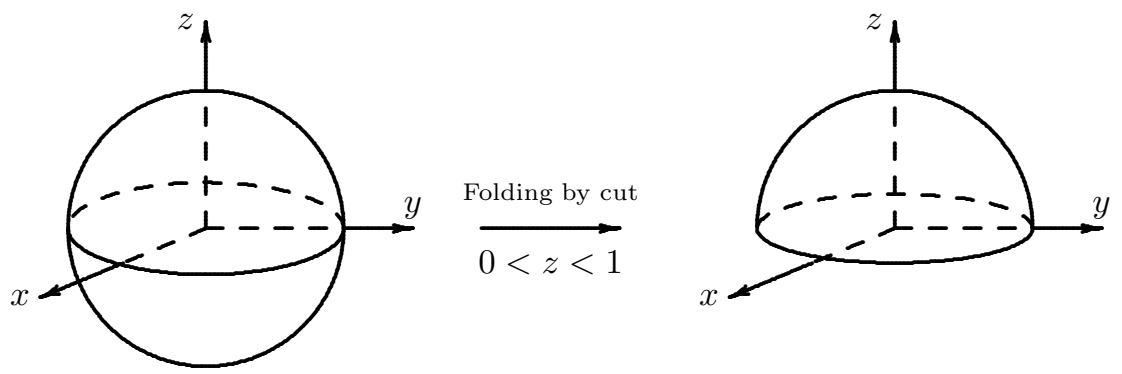

the result graph is open 2-manifold without boundary.

Case 5. If we take the boundary conditions in the form

$$
\begin{aligned}
x(0, \phi)=\sin \phi, & x(\pi, \phi)=-\sin \phi, \\
y(0, \phi)=0, & y(\pi, \phi)=0, \\
z(0, \phi)=\cos \phi, & z(\pi, \phi)=\cos \phi, \\
0<\theta<\pi, & 0 \leq \phi \leq \pi,
\end{aligned}
$$

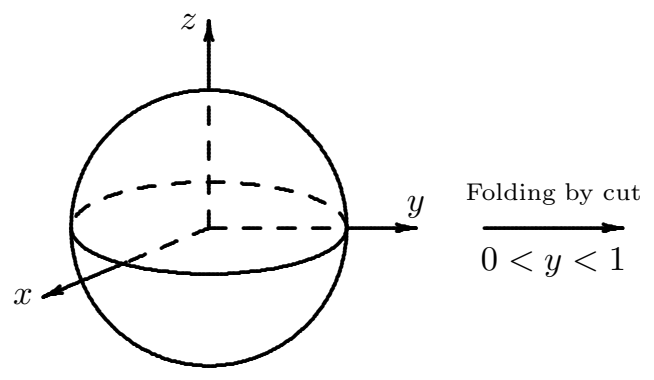

the result shape is open 2-manifold with boundary.

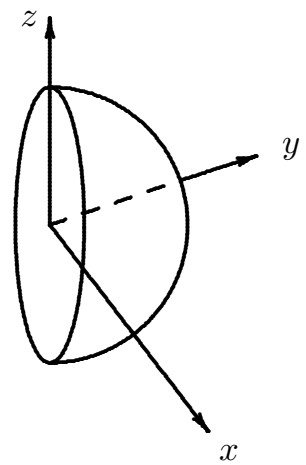

Note 1. From the boundary conditions of the Case $1-5$ we find that the limit of the foldings of the unit sphere is a circle (see Figure 4). 

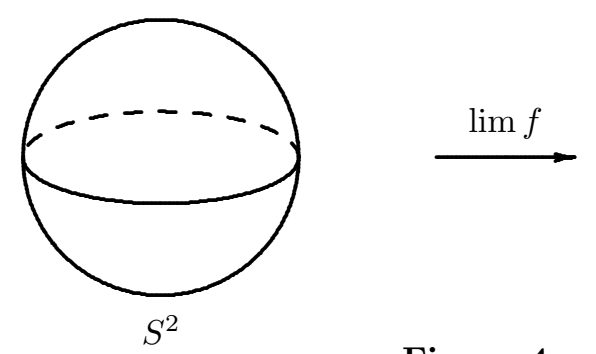

Figure 4.

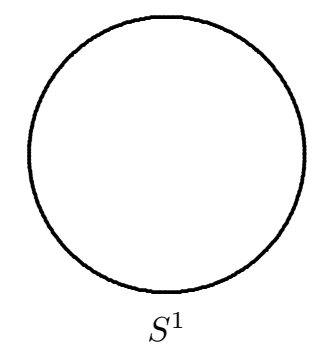

Case 6. If we take $-1<x<1,0<y<1, \frac{-1}{\sqrt{2}}<z<\frac{1}{\sqrt{2}}$ where $0<\theta<\pi$, $\frac{\pi}{4}<\phi<\frac{3 \pi}{4}$ we find that the shape after folding is 2 -manifold with boundary (see Figure $5)$.

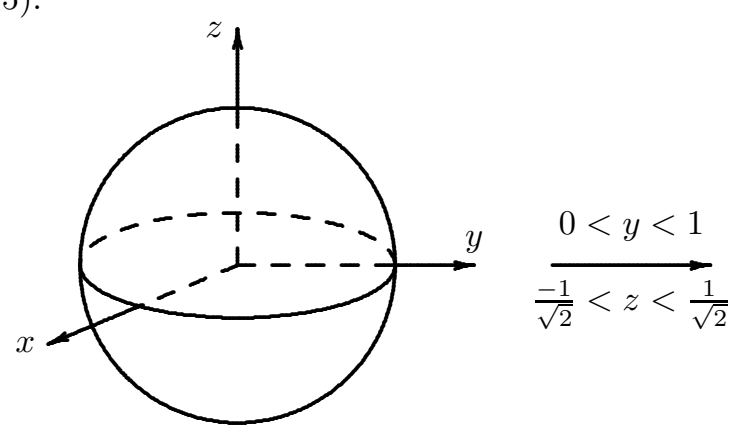

Figure 5.

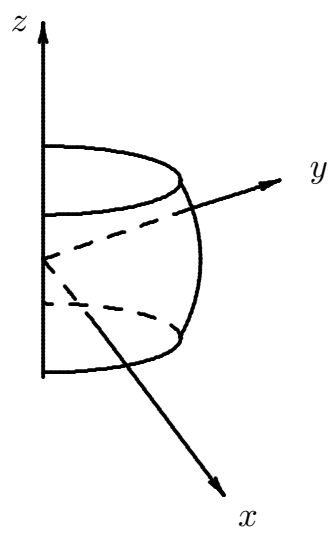

The above cases are not only the cases of foldings of $S^{2}$ but there are many cases also are foldings of $S^{2}$.

Note 2. The conditions of Case 6 represent the end of the limit foldings of the unit sphere $S^{2}$ which is a tow points (see Figure 6 ).

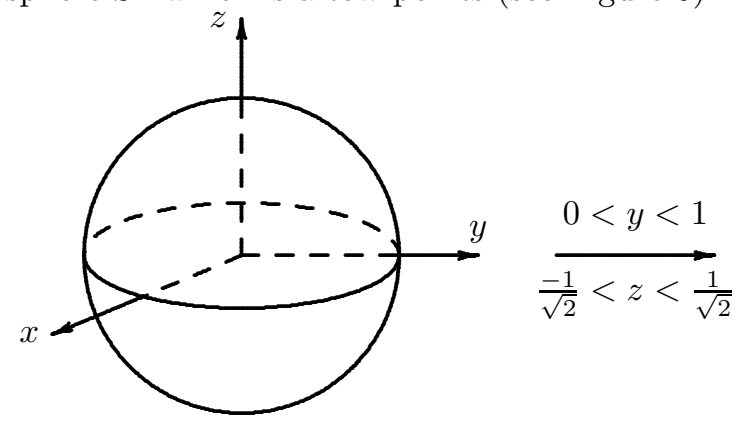

Figure 6. 
Note 3. We note from the Case $1-6$ that a restriction on one of the parameter $\theta$ or $\Phi$ is folding the sphere $S^{2}$ into a circle but a restriction on the two parameters $\theta$ and $\Phi$ is folding the sphere $S^{2}$ into two points.

Example 3. Consider the parametric equations of tours $T^{1}$ given by

$$
\begin{aligned}
& x_{1}=(b+a \cos u) \cos v, \quad 0<v<2 \pi, 0<u<2 \pi, \\
& x_{2}=(b+a \cos u) \sin v, \\
& x_{3}=a \sin u,
\end{aligned}
$$

the boundary-value partial differential equations of the tours $T^{1}$ are

$$
\begin{aligned}
& D_{V} x_{1}=-x_{2} \\
& D_{V} x_{2}=x_{1} \\
& D_{V} x_{3}=0 \\
& D_{u} x_{1}=a \sin u \cos (v+\pi) \\
& D_{u} x_{1}=a \sin u \sin (v+\pi) \\
& D_{u} x_{3}=a \cos u \\
& x_{1}(v, 0)=(b+a) \cos v \\
& x_{2}(v, 0)=(b+a) \sin v \\
& x_{3}(v, 0)=0 \\
& 0<v<2 \pi, \quad 0<u<2 \pi
\end{aligned}
$$

if we take the part

$$
X_{v}=\left[\begin{array}{ccc}
0 & -1 & 0 \\
1 & 0 & 0 \\
0 & 0 & 0
\end{array}\right] X
$$

the eigenvalues of the matrix $\left[\begin{array}{ccc}0 & -1 & 0 \\ 1 & 0 & 0 \\ 0 & 0 & 0\end{array}\right]$ are $\lambda_{1,2}= \pm i, \lambda_{3}=0$ which represent a limit of $\lim f_{n}\left\{T^{1}-S^{1}\right\}$ (see Figure 7 ). If we take $\left|\lambda_{1}\right|<1,\left|\lambda_{2}\right|<1$ the tours $T^{1}$ folding into itself and also if $\left|\lambda_{1}\right|>1,\left|\lambda_{2}\right|>1$ the tours $T^{1}$ unfolding into itself.

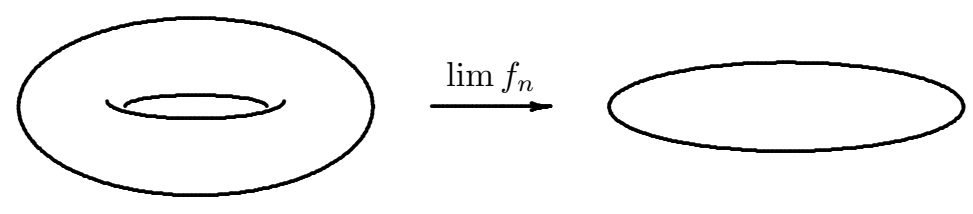

Figure 7. 
If we take the part

$$
\begin{aligned}
& D_{u} x_{1}=a \sin u \cos (v+\pi) \\
& D_{u} x_{1}=a \sin u \sin (v+\pi) \\
& D_{u} x_{3}=a \cos u
\end{aligned}
$$

we find it a type of folding.

Example 4. In this example we will show some of folding $T^{1}$ which corresponding to the cases in example two.

Case 1. If we take the boundary conditions in the form

$$
\begin{aligned}
& x_{1}\left(v, \frac{\pi}{2}\right)=b \cos v, \quad x_{1}\left(v, \frac{3 \pi}{2}\right)=b \cos v \\
& x_{2}\left(v, \frac{\pi}{2}\right)=b \sin v, \quad x_{2}\left(v, \frac{3 \pi}{2}\right)=b \cos v \\
& x_{3}\left(v, \frac{\pi}{2}\right)=a, \quad x_{3}\left(v, \frac{3 \pi}{2}\right)=-a, \\
& \text { Folding by cut }
\end{aligned}
$$
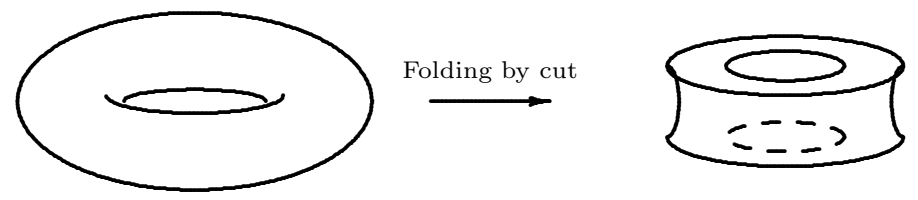

the result graph is 2-manifold.

Case 2. If we take the boundary conditions in the form

$$
\begin{array}{rlrl}
x_{1}(v, 0)= & (b+a) \cos v, & x_{1}(v, \pi)=(b-a) \cos v, \\
x_{2}(v, 0)= & (b+a) \sin v, & & x_{2}(v, \pi)=(b-a) \cos v, \\
x_{3}(v, 0)=0, & & x_{3}(v, \pi)=0 \\
& 0<u<\pi, & 0<v<2 \pi,
\end{array}
$$

the graph after folding by cut is the upper half of the tours $T^{1}$ which is 2-manifold.

Case 3. This case corresponding to the Case 4 in Example 2 the boundary condition are

$$
x_{1}(v, \pi)=(b-a) \cos v, \quad x_{1}(v, 2 \pi)=(b+a) \cos v,
$$




$$
\begin{array}{rlrl}
x_{2}(v, \pi)= & (b-a) \sin v, & & x_{2}(v, 2 \pi)=(b+a) \sin v, \\
x_{3}(v, \pi)=0, & & x_{3}(v, 2 \pi)=0, \\
& \pi<u<2 \pi, & & 0<v<2 \pi
\end{array}
$$

the graph after folding by cut is the upper half of the tours $T^{1}$ which is 2-manifold.

Case 4. This case corresponding to the Case 5 in Example 2 the boundary condition are

$$
\begin{array}{rlrl}
x_{1}(0, u)=b+a \cos u, & & x_{1}(2 \pi, v)=-(b+a \cos u), \\
x_{2}(0, u)=0, & & x_{2}(\pi, u)=0, \\
x_{3}(0, u)= & a \sin u, & & x_{3}(\pi, u)=a \sin u, \\
& 0<u<2 \pi, & & 0<v<\pi,
\end{array}
$$
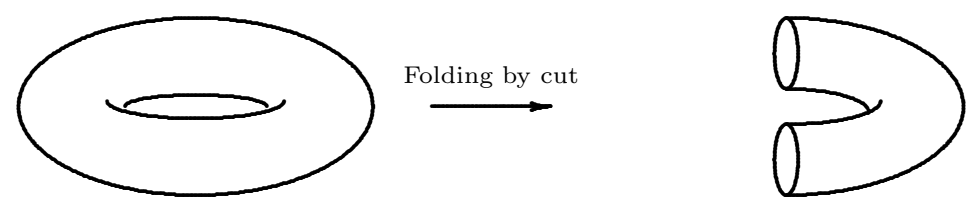

the graph after folding is 2-manifold with boundary.

Note 4. From the boundary conditions of the Case $1-4$ above we conclude that the limit folding of the tours $T^{1}$ is a circle (see Figure 7).

Case 5. If we make a restriction on $u$ and $v$ as $\frac{\pi}{2}<u<\frac{3 \pi}{2}, 0<v<\pi$ we have a folding by cut (see the following Figure 8).
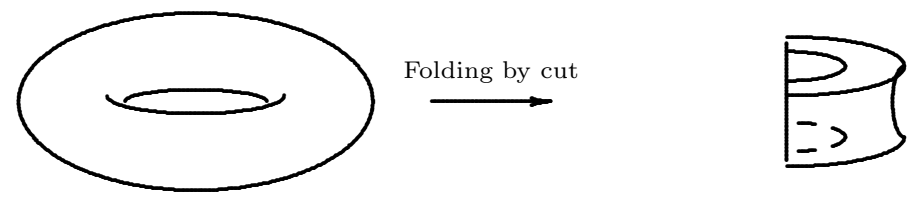

Figure 8 .

the result graph is 2 -manifold.

Note 5. There is a homeomorphism between folding of the unit sphere $S^{2}$ and folding of the tours $T^{1}$. 


\section{References}

[1] M. P. Docarmo, Differential Geometry of Curves and Surfaces, Englewood Ciff, New Jersey, 1976.

[2] M. El-Ghoul, Unfolding of graphs and uncertain graph, The Australian Senior Mathematics Journal Sandy Bay 7006 Tasmania Australia [accepted].

[3] M. El-Ghoul, The limit of folding of a manifold and its deformation retract, J. Egyptian Math. Soc. 5(1997), 133-140.

[4] M. El-Ghoul, Unfolding of Riemannian manifold, Commun Fac. Sci. Unversity of Ankara Ser. A 37(1988), 1-4.

[5] M. El-Ghoul, The deformation retract of the complex projective space and its topological folding, J. Mater Sci. 30(1995), 45-48.

[6] M. El-Ghoul, Fractional folding of manifold, Chaos, Solutions and Fractals 12(2001), 10191023.

[7] H. El-Hamouly and I. M. Mousa, Homoclinic bifpuraction of three dimensional system, Proceedings of the Fifth orma Conference, Military Technical College, Cairo, Egypt, 23-25, November (1993).

[8] P. DI. Francesco, Folding and coloring problem in Mathematics and Physics, Balliten of American Mathematic Society 37(2000), 251-307.

[9] S. T. Hu, Elements of General Topology, Holden-Dayinc, San Francisco, 1964.

[10] W. S. Massey, Algebraic Topology, An introduction, Harcourt Brace and world, New York, 1967.

[11] I. M. Mousa, On the bifurcation of ordinary differential equations of dimension greater than two, A Thesis of Ph.D. in Mathematics, Tanta Univ. Egypt, 1994.

[12] L. Perko, Differential Equations and Dynamical Systems, Springer-Verlag. New York, 1991.

[13] R. Seydel, From Equilibrium to Chaos, Elsevir, New York, Amesterdam, London, 1988.

[14] J. M. Singer and J. A. Thorpe, Lecture Notes on Elementary Topology and Geometry, Springer-Verlag, New York, 1967.

Department of Mathematics, Faculty of Science, Tanta University, Tanta, Egypt. 